\title{
Statistics of Advective Stretching in Three-dimensional Incompressible Flows
}

\author{
Natarajan Subramanian • Louise H. Kellogg • \\ Donald L. Turcotte
}

Received: 4 July 2008 / Accepted: 14 August 2009 / Published online: 9 September 2009

(C) The Author(s) 2009. This article is published with open access at Springerlink.com

\begin{abstract}
We present a method to quantify kinematic stretching in incompressible, unsteady, isoviscous, three-dimensional flows. We extend the method of Kellogg and Turcotte (J. Geophys. Res. 95:421-432, 1990) to compute the axial stretching/thinning experienced by infinitesimal ellipsoidal strain markers in arbitrary three-dimensional incompressible flows and discuss the differences between our method and the computation of Finite Time Lyapunov Exponent (FTLE). We use the cellular flow model developed in Solomon and Mezic (Nature 425:376-380, 2003) to study the statistics of stretching in a threedimensional unsteady cellular flow. We find that the probability density function of the logarithm of normalised cumulative stretching $(\log S)$ for a globally chaotic flow, with spatially heterogeneous stretching behavior, is not Gaussian and that the coefficient of variation of the Gaussian distribution does not decrease with time as $t^{-\frac{1}{2}}$. However, it is observed that stretching becomes exponential $\log S \sim t$ and the probability density function of $\log S$ becomes Gaussian when the time dependence of the flow and its three-dimensionality are increased to make the stretching behaviour of the flow more spatially uniform. We term these behaviors weak and strong chaotic mixing respectively. We find that for strongly chaotic mixing, the coefficient of variation of the Gaussian distribution decreases with time as $t^{-\frac{1}{2}}$. This behavior is consistent with a random multiplicative stretching process.
\end{abstract}

Keywords Kinematic mixing $\cdot$ Chaos $\cdot$ Multiplicative process

\section{Introduction}

Kinematic mixing in fluids is an important area of research and has wide ranging applications in fields like chemical engineering, materials science, geology, and geophysics. Kinematic mixing in a flow field neglects diffusive mixing. Significant work has been done to

This work was supported by a grant from the W.M. Keck Foundation and by award EAR-0810291 from the National Science Foundation.

N. Subramanian $(\bowtie) \cdot$ L.H. Kellogg · D.L. Turcotte

Geology Department, University of California, One Shields Avenue, Davis, CA 95616-8605, USA

e-mail: natarajan@geology.ucdavis.edu 
quantify kinematic mixing in two and three-dimensional flows. Some of the methods employed to study mixing include: theoretical studies on computing the stable and unstable manifolds of vortex ring flows [31, 33], theoretical [32, 39] and experimental [9] work on calculating advective flux as a measure of mixing in Taylor-Couette flows, theoretical work on quantifying mixing from stretching distributions $[25,30]$ of passive markers or power spectra of passive scalars [2] and numerous other theoretical [3, 13, 18, 25, 27, 28] and experimental $[7,8,13,18,27,28,37,38]$ tracer advection studies in two dimensional flows. Relatively fewer theoretical, [1, 11, 20, 29, 35] and experimental [11, 12, 16, 17, 21, 26, 35] tracer advection studies have been carried out in three-dimensional flows as well.

Mixing in fluids is achieved through stretching/thinning, stirring, folding, and diffusion of heterogeneities [26, 29]. Stretching increases the contact area between fluid elements, stirring transports these elements, while folding brings distant fluid elements into proximity, and diffusion helps local homogenization. Since in most known flows all these quantities are spatially and temporally heterogeneous, a complete treatise on mixing that involves all these parameters is currently unavailable. However, it can be argued that fast exponential stretching is a characteristic of efficient mixing [26]. Exponential stretching is generally associated with stagnation point flows. In this paper we compute the stretching in the three principal orthogonal directions associated with an ellipsoidal tracer as it moves in a 3D incompressible, unsteady flow.

Stretching of material lines or divergence of nearby tracer trajectories grows exponentially in chaotic flows. This exponential growth can be associated with a positive Lyapunov exponent. Lyapunov exponents are the time averaged measure of the separation of a set of perturbed orthogonal initial conditions. They provide a measure of chaos in dynamical systems [40]. A $n$-dimensional phase space has $n$ Lyapunov exponents characterising the $n$-orthogonal Lyapunov directions [40].

The distribution, self-similarity and multifractal properties of stretching fields and Lyapunov exponents have been well studied [4, 16, 17, 25, 27, 29] for 2D globally chaotic flows and those exhibiting regular elliptic islands in their Poincaré section. Relatively fewer studies exist for fully 3D flows. Consensus, however, does not exist on the asymptotic distribution of the Lyapunov exponents or stretching fields [4].

In this paper we develop a method to quantify stretching associated with the three principal axes of an infinitesimal strain ellipsoid. We then use our method to compute the stretching in the time-dependent, 3D cellular flow model developed in Solomon and Mezic [34]. We proceed to show that as the flow becomes more uniformly chaotic, the probability density function (PDF) of the logarithm of stretching exhibits a Gaussian behavior, implying that the most efficient stretching exhibits a log normal trend. The time evolution of the Gaussian behavior is then associated with a random multiplicative process. Based on our study we also suggest that only those flows which are both globally chaotic and exhibit similar Lagrangian dynamics throughout the flow domain will exhibit a linear increase in time of both the variance and the mean of stretching distribution.

\section{Deformation of a Ellipsoidal Inclusion}

Chaotic flows produce rapid stretching and thinning resulting in fast efficient mixing, while non-chaotic flows are comparatively slower. Hence to understand the nature and efficiency of mixing in complex flow fields, one has to understand the ability of the flow to stretch and thin passive inclusions. Here we extend the method of Kellogg and Turcotte [19] to compute the stretching associated with and orientation of the axes of ellipsoidal inclusions. 
Fig. 1 (Color online) The deformation of the ellipsoidal marker as it moves along its particle path

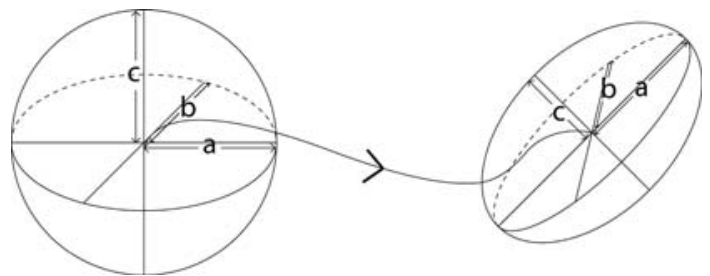

The deformation of elliptical strain markers has previously been considered in 2D viscous flows representing convection in Earths mantle and draws on a long history of work on deformation of markers in geological flows [6, 24].

To quantify the stretching associated with a chaotic flow, we introduce infinitesimal, passive, ellipsoidal strain markers into a specified cellular flow field. The characteristic length and velocity scales are used to non-dimensionalize the prescribed flow field. The threedimensional flow field is given as $\mathbf{U}(\mathbf{x}, t)$ in the Eulerian coordinate system $\mathbf{x}$. In order to carry out our analysis we introduce two new coordinates. First, we denote the position of the tracer by $\mathbf{X}$. The velocity of the fluid at the location of the tracer is then given by $\mathbf{U}(\mathbf{X}, t)$. Second, the coordinates defined by the principal axis a, b, and $\mathrm{c}$ of the ellipsoid are denoted by $\mathbf{X}^{\prime}$. The velocity field in this coordinate is responsible for the stretching and rotation of the ellipsoid and is denoted as $\mathbf{U}^{\prime}\left(\mathbf{X}^{\prime}, t\right)$.

Consider a fluid ellipsoidal inclusion defined by $\Phi\left(\mathbf{X}^{\prime}, t\right)=\mathrm{C}$ in a fluid matrix. Because the volume of the ellipsoid does not change, $\mathrm{C}$ is a constant. The "prime" superscript denotes a rotating frame in which the ellipsoid is defined. This frame rotates along-with the ellipsoid as it moves along its particle path and is aligned with the principal component axes as illustrated in Fig. 1. The kinematic boundary condition for the ellipsoid requires

$$
\frac{\partial \Phi}{\partial t}+\dot{\mathbf{X}}^{\prime} \cdot \nabla \Phi=0
$$

where

$$
\Phi=\mathbf{X}^{\prime T} G \mathbf{X}^{\prime}, \quad \mathbf{X}^{\prime} \in \mathbb{R}
$$

$\dot{\mathbf{X}}^{\prime}$ is the velocity of the ellipsoid and $G$ is the inverse square of the respective axes of the ellipsoid.

$$
G=\left(\begin{array}{ccc}
g_{11}(t) & 0 & 0 \\
0 & g_{22}(t) & 0, \\
0 & 0 & g_{33}(t)
\end{array}\right)
$$

where

$$
\begin{aligned}
& g_{11}(t)=\frac{1}{a(t)^{2}}, \\
& g_{22}(t)=\frac{1}{b(t)^{2}}, \\
& g_{33}(t)=\frac{1}{c(t)^{2}}
\end{aligned}
$$

$a(t), b(t), c(t)$ are the lengths of the principal axes at time $t$. Initially at $t=0$ we take $a_{0}>$ $b_{0}>c_{0}$. At later times, for the model flow considered in this paper, we find that this ordering 
of lengths is maintained so that $a(t)>b(t)>c(t)$. We define the cumulative stretching of the ellipsoidal inclusion by

$$
S(t)=\frac{a(t)}{a_{0}}
$$

For our chaotic mixing field we find that $S(t)$ has an exponential dependence on time so that

$$
\lambda=\frac{1}{t} \ln S
$$

is applicable. There is clearly a close association of $\lambda$ with the Lyapunov exponent. Any orthogonal coordinate system with origin at the center of the ellipsoid, and orientation parallel to the Eulerian coordinate system $\mathbf{X}$ can be made to coincide with a coordinate system defined by the axes of the ellipsoid through a rotation matrix. Thus, the fixed coordinates $\mathbf{X}$ is mapped to the rotating coordinates $\mathbf{X}^{\prime}$ through the rotation matrix $R$. The rotation matrix provides an orthogonal transformation from the fixed coordinates to the rotating coordinates.

$$
\begin{gathered}
\mathbf{X}^{\prime}=R \mathbf{X} \\
R=R_{x}(\psi) R_{y}(\theta) R_{z}(\phi)
\end{gathered}
$$

The angles $(\phi, \theta, \psi)$ are the rotations required about the coordinate system corresponding to $\mathbf{X}$, to align it with the coordinate system defined by the principal axes of the ellipsoid ( $\left.\mathbf{X}^{\prime}\right)$. The order of rotation about the axis is given by $z$-first, $y$-second, $x$-third. This rotation matrix $R(\phi, \theta, \psi)$ is given by,

$$
\left(\begin{array}{ccc}
\cos \phi \cos \theta & \cos \phi \sin \theta \sin \psi+\sin \phi \cos \psi & -\cos \phi \sin \theta \cos \psi+\sin \theta \sin \psi \\
-\sin \phi \cos \theta & -\sin \phi \sin \theta \sin \psi+\cos \phi \cos \psi & \sin \phi \sin \theta \cos \psi+\cos \phi \sin \psi \\
\sin \theta & -\cos \theta \sin \psi & \cos \theta \cos \psi
\end{array}\right)
$$

Differentiating (8) with respect to time we have

$$
\dot{\mathbf{X}}^{\prime}=R \dot{\mathbf{X}}+\dot{R} R^{T} \mathbf{X}^{\prime}
$$

which can be rewritten as

$$
R \dot{\mathbf{X}}=\dot{\mathbf{X}}^{\prime}-\dot{R} R^{T} \mathbf{X}^{\prime}
$$

Thus the velocity of the fluid in rotating coordinates is a function of its apparent velocity and a component related to the rate of rotation of the principal axes of the ellipsoid. Thus,

$$
\mathbf{U}^{\prime}=\dot{\mathbf{X}}^{\prime}+\Omega \mathbf{X}^{\prime}
$$

with $\Omega=R \dot{R}^{T}$ (since $R \dot{R}^{T}=-\dot{R} R^{T}$ ). Since we are using infinitesimal strain markers with the same material properties as the matrix, the velocity field of the fluid is not perturbed by the marker. We postulate that the motion takes a linear form and the velocity field can be written as a function of the strain rate tensor and position vector as

$$
\mathbf{U}=\dot{\mathbf{X}}=\Gamma \mathbf{X}
$$

and

$$
\mathbf{U}^{\prime}=\Gamma^{\prime} \mathbf{X}^{\prime}
$$


where $\Gamma$ and $\Gamma^{\prime}$ are the deformation gradient tensor in the fixed and rotating axes. The linear velocity field serves as a local approximation to a smooth, general velocity field.

$$
\Gamma^{\prime}=\left(\begin{array}{ccc}
\varepsilon_{11}^{\prime} & \varepsilon_{12}^{\prime}-\omega_{12}^{\prime} & \varepsilon_{13}^{\prime}+\omega_{13}^{\prime} \\
\varepsilon_{12}^{\prime}+\omega_{12}^{\prime} & \varepsilon_{22}^{\prime} & \varepsilon_{23}^{\prime}-\omega_{23}^{\prime} \\
\varepsilon_{13}^{\prime}-\omega_{13}^{\prime} & \varepsilon_{23}^{\prime}+\omega_{23}^{\prime} & \varepsilon_{33}^{\prime}
\end{array}\right)
$$

where $\varepsilon^{\prime}$ and $\omega^{\prime}$ are the strain rate and vorticity components respectively in the framework of the rotating axes and are given by,

$$
\begin{aligned}
& \varepsilon_{i j}^{\prime}=\frac{1}{2}\left(\frac{\partial u_{i}^{\prime}}{\partial X_{j}^{\prime}}+\frac{\partial u_{j}^{\prime}}{\partial X_{i}^{\prime}}\right), \\
& \omega_{12}^{\prime}=\frac{1}{2}\left(\frac{\partial v^{\prime}}{\partial X^{\prime}}-\frac{\partial u^{\prime}}{\partial Y^{\prime}}\right), \\
& \omega_{13}^{\prime}=\frac{1}{2}\left(\frac{\partial u^{\prime}}{\partial Z^{\prime}}-\frac{\partial w^{\prime}}{\partial X^{\prime}}\right), \\
& \omega_{23}^{\prime}=\frac{1}{2}\left(\frac{\partial w^{\prime}}{\partial Y^{\prime}}-\frac{\partial v^{\prime}}{\partial Z^{\prime}}\right)
\end{aligned}
$$

$u^{\prime}, v^{\prime}$ and $w^{\prime}$ are the components of the fluid velocity field $U^{\prime}$ in the rotating axes and,

$$
\Gamma=\left(\begin{array}{ccc}
\varepsilon_{11} & \varepsilon_{12}-\omega_{12} & \varepsilon_{13}+\omega_{13} \\
\varepsilon_{12}+\omega_{12} & \varepsilon_{22} & \varepsilon_{23}-\omega_{23} \\
\varepsilon_{13}-\omega_{13} & \varepsilon_{23}+\omega_{23} & \varepsilon_{33}
\end{array}\right)
$$

where $\varepsilon$ and $\omega$ are the strain rate and vorticity components respectively in the framework of the fixed axes and are given by,

$$
\begin{aligned}
& \varepsilon_{i j}=\frac{1}{2}\left(\frac{\partial u_{i}}{\partial X_{j}}+\frac{\partial u_{j}}{\partial X_{i}}\right), \\
& \omega_{12}=\frac{1}{2}\left(\frac{\partial v}{\partial X}-\frac{\partial u}{\partial Y}\right), \\
& \omega_{13}=\frac{1}{2}\left(\frac{\partial u}{\partial Z}-\frac{\partial w}{\partial X}\right), \\
& \omega_{23}=\frac{1}{2}\left(\frac{\partial w}{\partial Y}-\frac{\partial v}{\partial Z}\right)
\end{aligned}
$$

$u, v$ and $w$ are the components of the fluid velocity field $U$ in the fixed axes. The velocity gradient tensor in the rotating frame $\left(\Gamma^{\prime}\right)$ is related to velocity gradient tensor in the fixed frame $(\Gamma)$ by

$$
\Gamma^{\prime}=R \Gamma R^{T}+\dot{R} R^{T}
$$

Substituting (12) into (1) we obtain,

$$
\frac{\partial \Phi}{\partial t}+\mathbf{U}^{\prime} \cdot \nabla \Phi-\Omega \mathbf{X}^{\prime} \cdot \nabla \Phi=0
$$


Therefore, substituting (2) and (14) into (24) we obtain,

$$
\dot{G}+G \Gamma^{\prime}+\Gamma^{\prime T} G=\Omega G-G \Omega
$$

Equation (25) thus gives us the following six coupled ordinary differential equations (ODEs), which describe the evolution of the axes of the ellipsoid and the angles of rotation between the ellipsoidal axis and the fixed axes.

$$
\begin{gathered}
\dot{g}_{11}+2 g_{11} \varepsilon_{11}^{\prime}=0, \\
\dot{g}_{22}+2 g_{22} \varepsilon_{22}^{\prime}=0, \\
\dot{g}_{33}-2 g_{33}\left(\varepsilon_{11}^{\prime}+\varepsilon^{\prime}{ }_{22}\right)=0, \\
\left(g_{11}+g_{22}\right) \varepsilon_{12}^{\prime}+\left(g_{22}-g_{11}\right) \omega_{12}^{\prime}=0, \\
\left(g_{11}+g_{33}\right) \varepsilon_{13}^{\prime}+\left(g_{11}-g_{33}\right) \omega_{13}^{\prime}=0, \\
\left(g_{33}+g_{22}\right) \varepsilon_{23}^{\prime}+\left(g_{33}-g_{22}\right) \omega_{23}^{\prime}=0
\end{gathered}
$$

Therefore, given $\Gamma$ in the fixed coordinates, one can compute $\Gamma^{\prime}$ for the rotating coordinates and hence the evolution of the axes of the ellipsoid. The above equations give us a simple and robust formulation for computing the stretching/thinning associated with ellipsoidal tracers in three-dimensional flows. It must be noted that the method can be used for steady as well as unsteady flows.

The time averaged measure of the stretching experienced by the strain ellipsoid, in the three orthogonal directions, is equivalent to the three Lyapunov exponents of the flow computed at the starting point of the tracer, however, not identical. Computing the stretching experienced by the tracer in the strain basis provides a much sharper picture of the underlying dynamics of the flow than computing the Lyapunov exponent which is simply the maximal stretching. To demonstrate this we have shown the stretching map calculated for three grids of $212 \times 212$ tracers starting in the $x=0, y=2 \pi$ and $z=2 \pi$ plane, respectively, for the $A B C$ flow at time $t=-8$. The parameters $A, B, C$ were chosen as $\sqrt{3}, \sqrt{2}, 1$ respectively. The particular time and parameters were chosen to make a comparison with the work of Haller [15]. The $t=-8$ stretching map shown in Fig. 2 is different than the Lyapunov exponent map show in Fig. 6 of Haller [15]. As Haller [15] points out, the direct Lyapunov exponent map does not bring out the resonant tori visible in the Type 7 hyperbolicity plot (shown in Fig. 6 of Haller [15]), in our method, however, it is clearly visible (shown in Fig. 2). While it has been pointed out that using the eigenvectors of the strain rate tensor, as basis, to locate hyperbolic structures, in general, is not advantageous [14, 22], the same cannot be said for computing the stretching of the infinitesimal tracers in the strain basis. In the latter case, using the strain basis is necessary in order to evaluate the stretching experienced by the ellipsoid in its current configuration.

Typically to compute the Lyapunov exponent associated with a flow, one computes the square root of the eigenvalues of the discrete, left or right, Cauchy-Green tensor at various points in the flow at prescribed times. In order to evolve the components of the CauchyGreen tensor through time, one could either compute the time evolution of the deformation gradient tensor (by using the velocity gradient tensor) [24] or use a flow map [15]. If the velocity gradient tensor is used to compute the components of the Cauchy-Green tensor (by forming the deformation gradient tensor), 12 ODEs per tracer per time step would have to be solved as opposed to solving 9 ODEs per tracer per time step ( 3 equations of motion and equations 26-31) by our method. Alternatively using the method suggested by [15] 
Fig. 2 (Color online) The backward time stretching map for the $A B C$ flow, $A=\sqrt{3}, B=\sqrt{2}$, $C=1$. A grid of $212 \times 212$ strain ellipsoids was used to compute the stretching map. The resonant tori [10] are clearly visible as light green regions

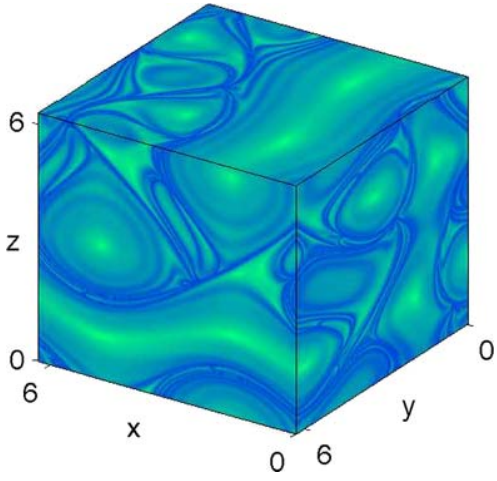

requires computing 9 spatial gradients (preferably centered differenced) and solving 3 ODEs at each time step. Irrespective of which of these two methods is used to compute the discrete Cauchy-Green tensor our method is neither more expensive nor more complex.

Also note that the strain marker is placed into a model of physical, not phase space; it is a marker in a fluid flow, not a tracer following the evolution of a set of equations describing a complex dynamical system. The equations describing a chaotic dynamical system may have any number of degrees of freedom, with the corresponding number of Lyapunov exponents. A simple example would be the classical Lorenz equations [23], with 3 degrees of freedom; that system of equations approximates convection behavior in the atmosphere in an extremely truncated model. In contrast, whatever the number of degrees of freedom of the equations governing the flow, a strain marker in an incompressible fluid has 6 degrees of freedom: the 3 axes of its principal components, together with the 3 directions that describe the orientation of the principal axes of the strain ellipsoid. We place the infinitesimal strain marker in the fluid, and then calculate the resulting strain (the evolution of the principal axes) and change in orientation. Because volume is conserved, the change in length of each principal axis is related to the other principal axes. As one axes lengthens, another must shorten; the result may be a nearly spherical ellipsoid (with little cumulative deformation), a cigar shape (in which one axis grows exponentially), or a pancake (in which two axes grow). Also the strain ellipsoid can experience exponential stretching and thinning even in a flow that is not chaotic. An example would be the "pure shear" case, in which a strain ellipsoid is placed in a stagnation point with steady, continuous stretching on one set of axes and steady thinning in an orthogonal direction. Computing deformation of strain markers reflects the processes that lead directly to mixing by determining the rate of increase of the surface area of heterogeneities.

In spite of the close association between the stretching experienced by the strain ellipsoid and the Lyapunov exponent in fluid flows, keeping in mind the physical significance of stretching, the rest of this manuscript discusses the stretching properties of the flow rather than the Lyapunov exponent.

\section{Deformation of Markers in a Cellular Flow}

In this section we study the statistics of stretching of infinitesimal ellipsoidal markers in the three-dimensional unsteady flow introduced by [34]. The velocity field in their cellular flow model is given by

$$
u=-\cos \left(\pi x_{s}(t)\right) \sin (\pi y)+\varepsilon \sin \left(2 \pi x_{s}(t)\right) \sin (\pi z),
$$


Table 1 The parameter values and a brief description of the three different models considered

\begin{tabular}{|c|c|c|c|c|}
\hline Model number & $\omega$ & $\varepsilon$ & $b$ & Description \\
\hline Model 1 & 2.5 & 0.005 & 0.01 & $\begin{array}{l}\text { Weakly 3D and time-dependent. Driving frequency is } \\
\text { resonant with circulation frequency }\end{array}$ \\
\hline Model 2 & 4.0 & 0.005 & 0.01 & $\begin{array}{l}\text { Weakly } 3 \mathrm{D} \text { and weakly time-dependent. Driving frequency is } \\
\text { not resonant with circulation frequency }\end{array}$ \\
\hline Model 3 & 2.5 & 0.01 & 0.09 & $\begin{array}{l}\text { Weakly } 3 \mathrm{D} \text { and moderately time-dependent. Driving frequency is } \\
\text { resonant with circulation frequency }\end{array}$ \\
\hline Model 4 & 2.5 & 1.000 & 1.00 & Strongly 3D and strongly time-dependent \\
\hline
\end{tabular}

$$
\begin{aligned}
& v=\sin \left(\pi x_{s}(t)\right) \cos (\pi y)+\varepsilon \sin (2 \pi y) \sin (\pi z), \\
& w=2 \varepsilon \cos (\pi z)\left[\cos \left(2 \pi x_{s}(t)\right)+\cos (2 \pi y)\right], \\
& x_{s}(t)=x+b \sin \omega t
\end{aligned}
$$

where $b$ and $\omega$ represent the dimensionless oscillation amplitude and frequency and the 3D component of the flow is characterized by $\varepsilon$. The flow is confined to the region $-0.5 \leq$ $y \leq 0.5$ and $-0.5 \leq z \leq 0.5$ and the boundaries are free slip (zero shear). The flow in the $x$-direction is periodic but time-dependent. As pointed out by [34], the flow considered in this paper emulates properties exhibited by laminar vortex flows. Since vortical flows are commonly encountered in natural processes, studies done here must be widely applicable. Also, the flow has been well studied $[34,36]$ and allows for independent control of the amplitude of time dependence and three-dimensionality of the velocity field, which allows us to study their relative controls on stretching and hence mixing.

In this paper we consider a suite of models with varying $\varepsilon$ and $b$. We present graphs and figures for four models with the parameter values $\omega, \varepsilon$, and $b$ given in Table 1 . The models represent,

1. Model $1(\omega=2.5, \varepsilon=0.005$ and $b=0.01)$ : Is weakly three-dimensional and timedependent and resembles a vortical flow bounded by solid boundaries (example of particle path shown in Fig. 3(a)). The circulation frequency is resonant with the driving frequency and results in weakly chaotic mixing.

2. Model $2(\omega=4.0, \varepsilon=0.005$ and $b=0.01)$ : Similar to Model 1 , this model is also weakly three-dimensional and time-dependent and resembles a vortical flow bounded by solid boundaries. However, unlike Model 1, the circulation frequency is not resonant with the driving frequency. This difference leads to the presence of regular elliptic islands in the flow and results in inefficient mixing.

3. Model $3(\omega=2.5, \varepsilon=0.01$ and $b=0.09)$ : Is moderately three-dimensional and timedependent and still resembles a vortical flow bounded by solid boundaries. The circulation frequency is resonant with driving frequency. This model is more three-dimensional and time dependent than Models 1 and 2 and lies on the transition between weak chaotic mixing and strong chaotic mixing.

4. Model $4(\omega=2.5, \varepsilon=1.0$ and $b=1.0)$ : Is strongly three-dimensional and timedependent. The particle path topology is significantly different from Models 1, 2, and 3 , the particle paths do not lie on toric surfaceslike the previous models (example of particle path shown in Fig. 3(b)). This model exhibits strong chaotic mixing. 
(a)

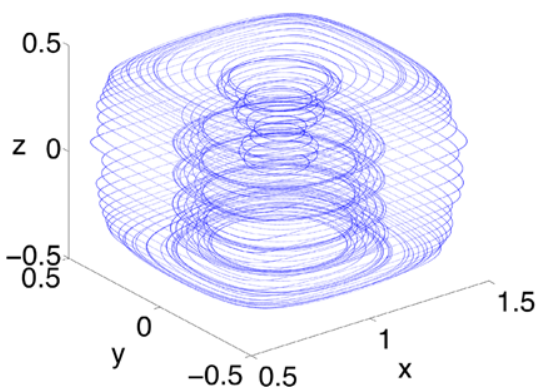

(b)

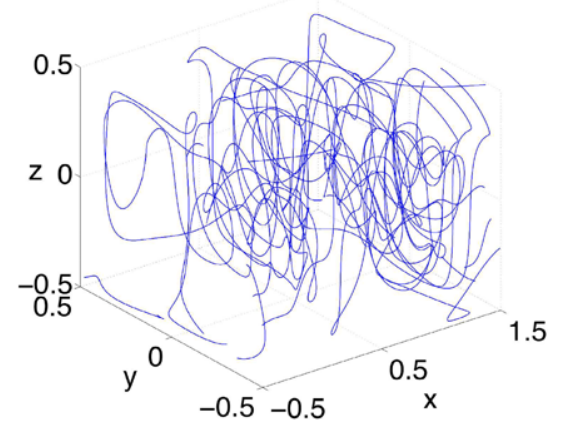

Fig. 3 (Color online) The three-dimensional particle paths of a tracer initially at $(1.1,0,0)$. (a) $\omega=2.5, \varepsilon=0.005$ and $b=0.01$. (b) $\omega=2.5, \varepsilon=1$ and $\mathrm{b}=1$. Note the vortex nature of the path on the left and the lack thereof on the right. Increasing three-dimensionality and time dependence breaks down the vortex structure of the flow, particle paths start wandering in the domain

The first two models were considered previously [34] and are weakly three-dimensional and time-dependent. The case $\omega=2.5$ exhibits chaotic mixing, whereas $\omega=4$ shows inefficient mixing due to the presence of regular elliptic islands, however, in both models particle paths are confined to toric surfaces. These authors attribute the uniform mixing of Model 1 to a resonance between the driving frequency and the circulation frequency of the flows. The third model is weakly three-dimensional and moderately time-dependent. The strength of the three-dimensional component of the velocity field is twice as strong and the amplitude of time dependence nine times as strong compared to Models 1 and 2. The fourth model is strongly $3 \mathrm{D}$ and time-dependent $(\varepsilon=1, b=1)$, there is complete breakdown of structure and the flow is uniformly globally chaotic. The four models were chosen to show the differences in the stretching distributions as the flow tends towards uniform global chaos. In particular, the three Models 1, 3, and 4 show the gradual transformation from weak chaotic mixing to strong chaotic mixing. In the following section we present the results of our stretching calculations for the four different models. We then proceed to describe the statistics of the PDF (probability density function) of the logarithm of stretching.

The non-dimensional box aspect ratio is $2 \times 1 \times 1$ in the $x \times y \times z$ directions. We place 500 ellipsoidal tracers along the line $(-0.5,0,0) \times(1.5,0,0)$ spaced 0.004 apart. The stretching and orientation of the principal axes are calculated from (26)-(31) as the ellipsoid moves along its particle path. The ellipsoidal tracers are initially roughly circular, the difference between axes being $1 \%$. Since the marker is infinitesimal, strain rate and vorticity at each step are calculated assuming the tracer is a point. The integration for both particle tracing and extension calculation is done using a step-adaptive fifth order Runge-Kutta method. The results shown below are for the normalized stretching of the maximum principal axis $\left(S=\frac{a}{a_{0}}\right)$.

\section{Stretching Results}

Time-dependent calculations were carried out to determine the cumulative stretching of each tracer $S$ as a function of time $t$. Typical examples of the time evolution of the three principal axes of an ellipsoidal tracer particle are given in Fig. 4 for Models 1 and 4 . The maximum principal axis $S=\frac{a}{a_{0}}$ grows nearly exponentially ( $\log _{10}$ is used), the minimum principal 
(a)

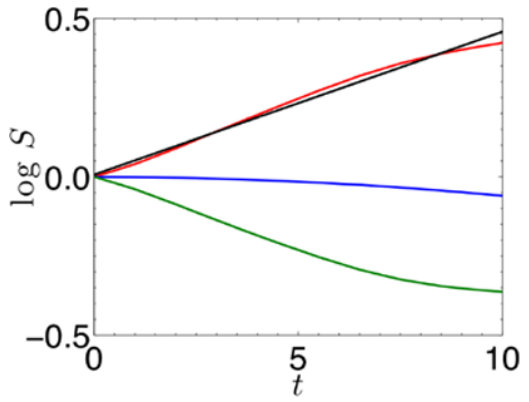

(b)

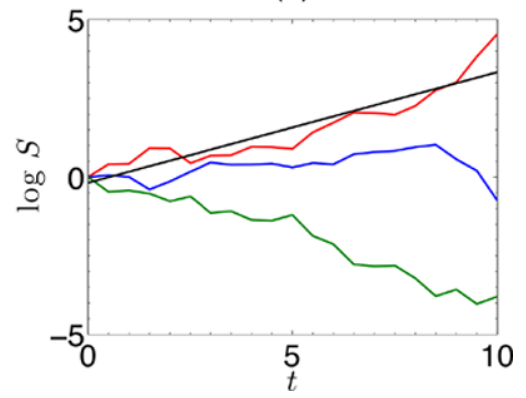

Fig. 4 (Color online) Stretching, defined as $\log S$, for the three ellipsoidal axes are plotted as a function of time $t$ for Models 1 and 4 . The tracer was initially located at $(1.1,0,0)$ in both models. In both figures the red line indicates the fastest growing axis $\left(\frac{a}{a_{0}}\right)$, blue line the intermediate axis $\left(\frac{b}{b_{0}}\right)$, and green line the shortening axis $\left(\frac{c}{c_{0}}\right)$. The black line is the best fit line for the stretching of the maximum principal axis. (a) Model $1 \omega=2.5, \varepsilon=0.005, b=0.01$. Slope of the best fit line is 0.045 and the value of $\lambda$, given by (7), is 0.104 . (b) Model $4 \omega=2.5, \varepsilon=1, b=1$. Slope of the best fit line is 0.356 and the value of $\lambda$, given by (7), is 0.820

(a)

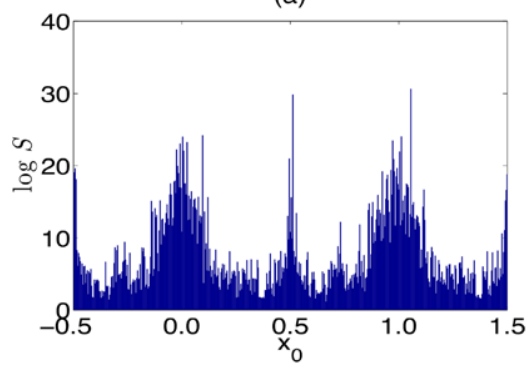

(b)

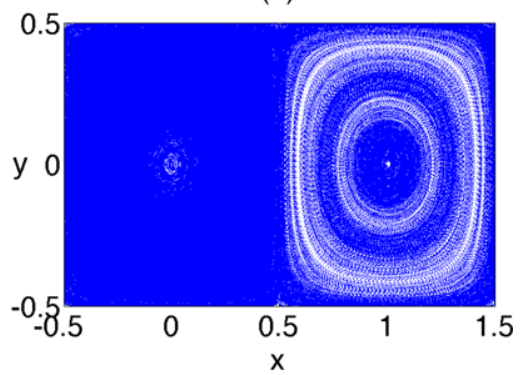

Fig. 5 (Color online) Stretching and Poincaré section for Model $1(\omega=2.5, \varepsilon=0.005$ and $b=0.01)$. (a) The cumulative stretching $\log S$ at $t=400$ is given as a function of the initial position $x_{0}$ of the tracers. (b) Poincaré section of the $z=0$ crossing of a single tracer with initial position $(1.1,0,0)$ is shown. In this and subsequent figures, we consider only the stretching of the maximum principal axis, $S=\frac{a}{a_{0}}$

axis $S=\frac{c}{c_{0}}$ decreases nearly exponentially, and the intermediate principal axis $S=\frac{b}{b_{0}}$ is nearly constant. We find that stretching in Model 4 is $\sim 8$ times stronger than in Model 1. The exponential stretching rate given by $(7)$, for a test tracer starting at $(1.1,0,0)$ is found to be $\lambda=0.104$ for Model 1 and $\lambda=0.820$ for Model 4 . This difference in the stretching rate of the test tracer between the two models is a result of the strong chaos present in Model 4 compared to Model 1.

In Figs. 5(a)-8(a) we give the cumulative stretching $\log S$ at a specified time as a function of the initial position of the tracers for the four models. In Figs. 5(b)-8(b) the Poincare sections of $z=0$ crossings of a single tracer with initial position $(1.1,0,0)$ are shown for the four models.

For Models 1 and 2 the stretching in chaotic regions (peaks in Figs. 5(a), 6(a) and filled regions in Figs. 5(b), 6(b)) is much higher than the regular regions. The stretching for Model $1(\omega=2.5, \varepsilon=0.005$ and $b=0.01)$ is more uniform compared to Model 2 $(\omega=4.0, \varepsilon=0.005$ and $b=0.01)$. Model $3(\omega=2.5, \varepsilon=0.01$, and $b=0.09)$, shown in 
(a)

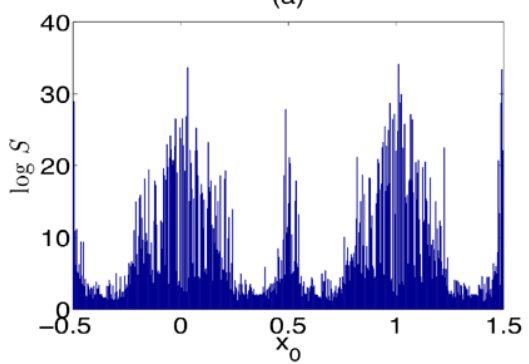

(b)

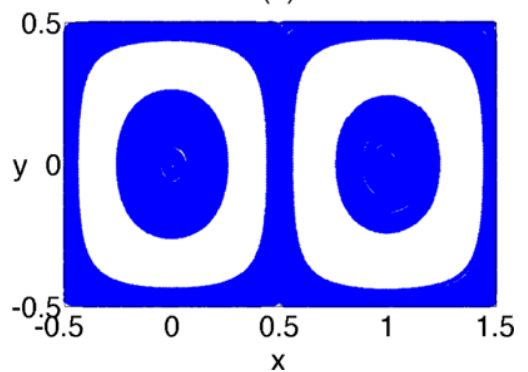

Fig. 6 (Color online) Stretching and Poincaré section for Model $2(\omega=4, \varepsilon=0.005$ and $b=0.01)$. Symbols are the same as in Fig. 5

(a)

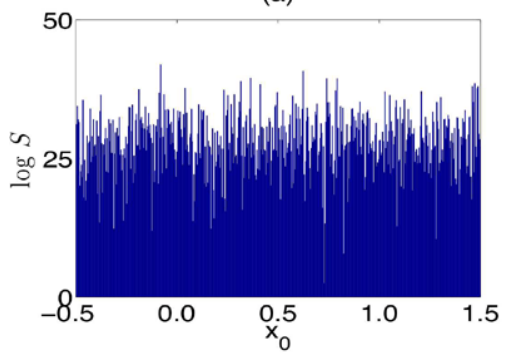

(b)

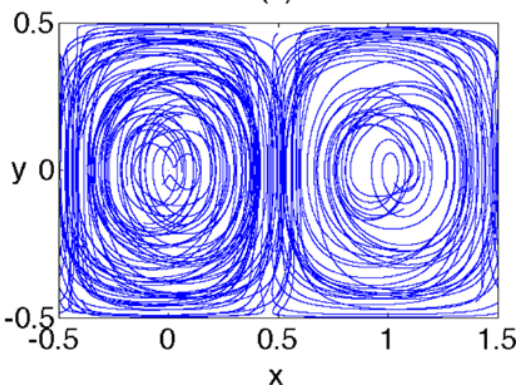

Fig. 7 (Color online) Stretching and Poincaré section for Model $3(\omega=2.5, \varepsilon=0.01$ and $b=0.09)$. Symbols are the same as in Fig. 5
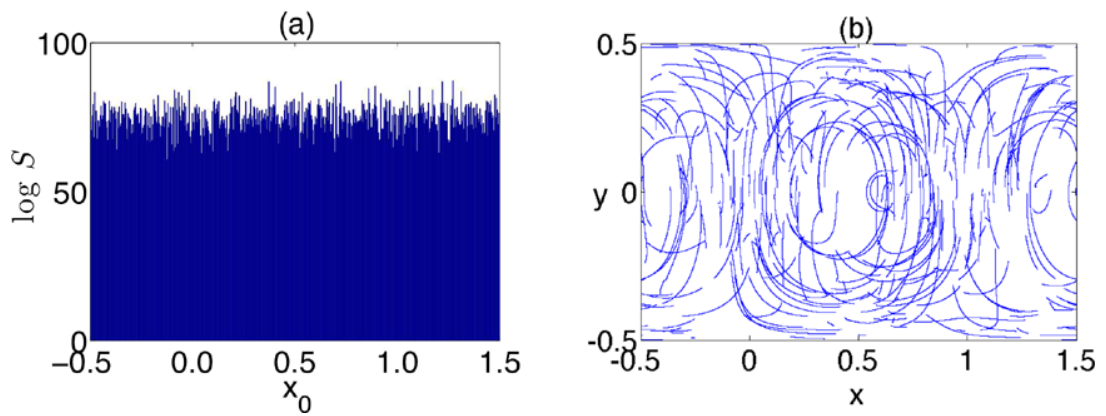

Fig. 8 (Color online) Stretching and Poincaré section for Model $4(\omega=2.5, \varepsilon=1$ and $b=1)$. Symbols are the same as in Fig. 5

Fig. 7, exhibits more uniform stretching than either Model 1 or 2 . In Model $4(\omega=2.5, \varepsilon=1$ and $b=1$ ) the stretching is very uniform and significantly greater than the previous three models as shown in Fig. 8. This is due to the strong three-dimensional component and time dependence of the flow (higher values of $\varepsilon$ and $b$ ). In the next section we look at the PDFs associated with $\log S$. 
Table 2 The slope of the mean stretching vs time plot (black line in the PDF plots) and the mean stretching rate is given for all the models at time $t=200$. Models 1 to 4 are the models for which we show graphs and carry out a detailed discussion. Models 5 to 13 were designed to analyze the effect of time dependence and three-dimensionality on stretching distributions

\begin{tabular}{llllll}
\hline Model number & $\omega$ & $\varepsilon$ & $b$ & Slope & $\lambda$ \\
\hline Model 1 & 2.5 & 0.005 & 0.01 & 53.282 & 0.044 \\
Model 2 & 4.0 & 0.005 & 0.01 & 42.986 & 0.053 \\
Model 3 & 2.5 & 0.01 & 0.09 & 7.3693 & 0.313 \\
Model 4 & 2.5 & 1.00 & 1.00 & 2.6875 & 0.860 \\
Model 5 & 2.5 & 0.01 & 0.01 & 45.679 & 0.051 \\
Model 6 & 2.5 & 0.01 & 0.03 & 21.841 & 0.110 \\
Model 7 & 2.5 & 0.01 & 0.05 & 9.814 & 0.240 \\
Model 8 & 2.5 & 0.01 & 0.07 & 8.059 & 0.290 \\
Model 9 & 2.5 & 0.005 & 0.02 & 28.242 & 0.080 \\
Model 10 & 2.5 & 0.015 & 0.02 & 31.873 & 0.071 \\
Model 11 & 2.5 & 0.025 & 0.02 & 23.876 & 0.097 \\
Model 12 & 2.5 & 0.035 & 0.02 & 21.854 & 0.106 \\
Model 13 & 2.5 & 0.045 & 0.02 & 22.084 & 0.104 \\
\hline
\end{tabular}

\section{Probability Distributions}

In this section we study the statistical distribution of values of $\log S$ for the 500 tracer particles at a specified time for our four models. In addition, we also present the results from several other models in order to attempt to isolate the effects of time- and spatial-dependence on the stretching behavior of the flow. If the values of $\log S$ satisfy a Gaussian distribution then the values of $s$ have a lognormal distribution. As noted by Arratia and Gollub [4] a consensus does not exist on the asymptotic distribution of the stretching $\log S$. Some investigations $[17,29]$ suggest that the asymptotic distribution of $\log S$ should be Gaussian, however, the results of Arratia and Gollub [4] do not show this behavior. In this section we present evidence that the asymptotic stretching distribution of $\log S$ in strongly chaotic flows tends towards a Gaussian as the flow becomes more three-dimensional and time-dependent. We also show that this shift towards a more Gaussian like distribution is accompanied by a decrease in slope in the $\overline{\log S}$ (where $\overline{\log S}$ is the $\operatorname{logarithm}$ of the mean stretching) versus time $(t)$ plot. A significant amount of work has been done on the self similarity of the stretching distributions $[4,16,17,25,29]$ and we do not discuss this further.

The PDFs of $\log S$ for the 500 particles are shown in Figs. 9-10 and 12-13, for models 1 to 4 respectively. The PDFs are plotted at their corresponding times. The PDF at time $t=50$ is normalized to 50. PDFs at later times are plotted at their corresponding time and normalized based on the ratio of the amplitude of their peak to that at time $t=50$. The lighter shades of green indicate increasing time. For all the models, the darkest shade of green corresponds to $t=50$ and the lightest shade corresponds to $t=200$. The intermediate values correspond to $t=100$ and $t=150$. The mean values of the stretching distribution at various times are given as red dots. The straight line indicates the best fit line to the mean stretching rate. The slope of this line is used to determine the value of $\lambda$ given in Table 2 .

From Figs. 9 and 10 we see that models 1 and 2 with $\omega=2.5$ and $\omega=4$ for $\varepsilon=0.005$ and $b=0.01$ show a strong peak in the lower end of the stretching spectrum. The value of $\log S$ for the primary peak in $\omega=2.5$ is an order of magnitude higher than that for $\omega=4$. From Fig. 11, we also see that both models show a weaker high end peak in the stretching spectrum at later times. The peak for $\omega=4.0$ is broader compared to the peak for $\omega=2.5$. We see that for both models with weak three-dimensionality and time dependence, the stretching is very asymmetric. Also, from Table 2 we see that the mean stretching rate 


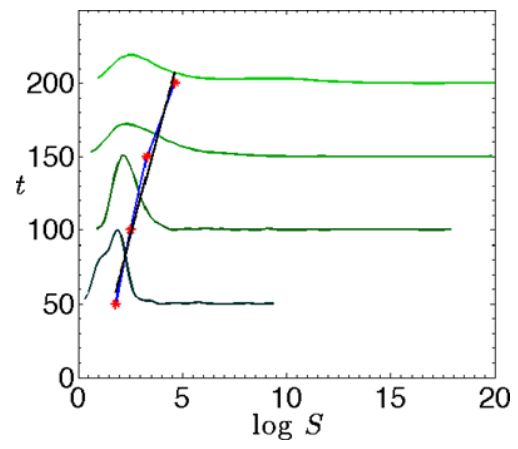

Fig. 9 (Color online) Model $1 \omega=2.5, \varepsilon=0.005$ and $b=0.01$. The PDFs of the cumulative stretching $\log S$ for the 500 tracer particles are given for various times. Lighter shades of green indicate further ahead in time. Time $t$ goes from $t=50$ (darkest green) to $t=200$ (lightest green). The intermediate values correspond to $t=100$, and $t=150$. The PDF curves are plotted at their corresponding times indicated on the $t$ axis. The peak height of the PDF at time $t=50$ is normalised to 50. Peak heights at other times are normalised based on the ratio of the peak intensity at that time to that at time $t=50$. The black line indicates the best fit line to the mean stretching vs time plot. The slope of the line is 53.282 and the mean stretching given by $\lambda=$ $\frac{1}{\text { slope }} \times \ln 10=0.044$

Fig. 10 (Color online) Model 2 $\omega=4.0, \varepsilon=0.005$ and $b=0.01$. Symbols and description are similar to Fig. 9. The slope of the line is 42.986 and the mean stretching rate given by $\lambda=$ $\frac{1}{\text { slope }} \times \ln 10=0.053$

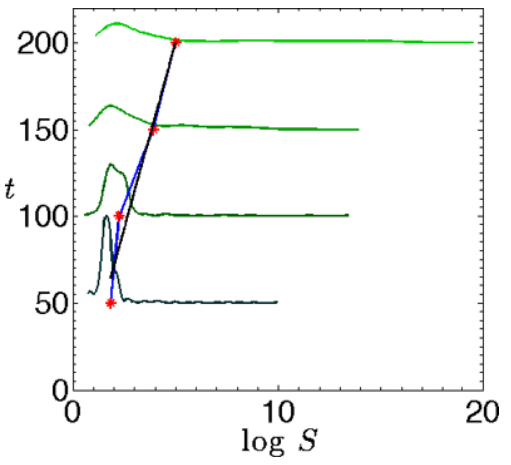

for Model $2(\lambda=0.053)$ is higher than the mean stretching rate for Model $1(\lambda=0.044)$, as a consequence of the broad high end peak in Model 2.

Model 3 given in Fig. 12 with $\omega=2.5, \varepsilon=0.01$, and $b=0.09$ exhibits stronger and more uniform stretching compared to Models 1 and 2. The mean stretching rate for Model 3 is $\lambda=0.313$. The secondary high end peak observed in the stretching spectrum of Models 1 and 2 is absent in Model 3. From Table 2, we see that as $\varepsilon=0.01$ is held constant and $b$ gradually increased from 0.01 to 0.09 (Model 3 and Models 5 to 8) the mean stretching rate increases. This monotonic increase in mean stretching rate is not observed when $b$ is held constant at 0.02 and $\varepsilon$ increased from 0.005 to 0.045 in Models 9 to 13. This indicates that $b$, the time dependence amplitude, is a stronger control on mean stretching and hence mixing for the flow considered in this paper.

Model 4 given in Fig. 13 with $\omega=2.5, \varepsilon=1.0$, and $b=1.0$ exhibits significantly stronger stretching compared to models 1 to 3 . The mean stretching rate for Model 4 is $\lambda=0.860$. The enhanced stretching rate in Model 4 is a result of the strong threedimensionality and time dependence of the flow. To show that PDFs of $\log S$ are Gaussian distributed, in Fig. 14 we show a plot of PDFs of $\log S$ at various times $(t=50,100,150$, 


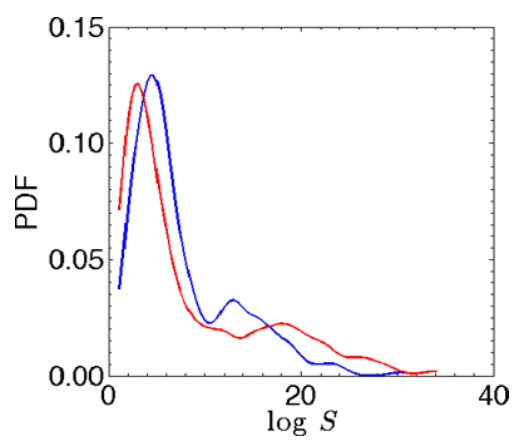

Fig. 11 (Color online) The PDFs of the cumulative stretching $\log S$ for the 500 tracer particles are given at time $t=400$ for two values of $\omega$. The blue curve corresponds to Model $1(\omega=2.5, \varepsilon=0005$ and $b=0.01)$ and the red curve corresponds to Model $2(\omega=4.0, \varepsilon=0005$ and $b=0.01)$. Secondary peaks are present in both models and the secondary peak for $\omega=4.0$ is wider than the secondary peak for $\omega=2.5$

Fig. 12 (Color online) Model 3 $\omega=2.5, \varepsilon=0.01$ and $b=0.09$. Symbols and description are similar to Fig. 9. The slope of the line is 7.3693 and the mean stretching rate given by $\lambda=$ $\frac{1}{\text { slope }} \times \ln 10=0.313$

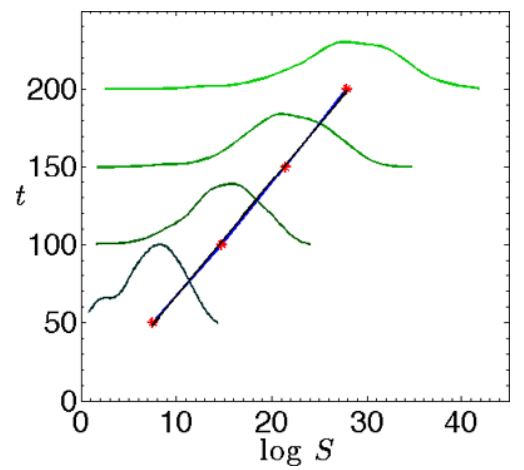

Fig. 13 (Color online) Model 4 $\omega=2.5, \varepsilon=1$ and $b=1$. Symbols and description are similar to Fig. 9. The slope of the line is 2.6875 and the mean stretching rate given by $\lambda=\frac{1}{\text { slope }} \times \ln 10=0.860$

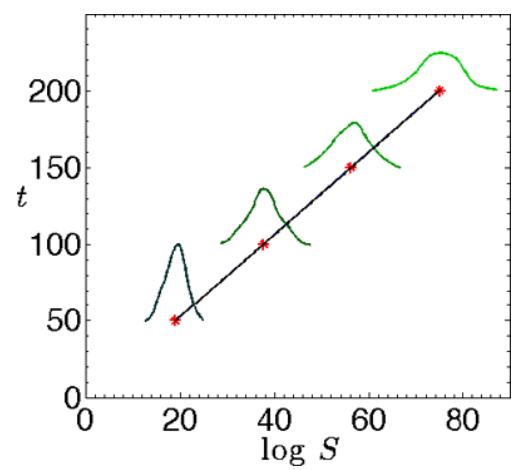

and 200) along with a quantitative Gaussian fit shown as a red curve. As can be seen, there is excellent agreement between the fit and the data. This lognormal distribution of $\log S$ is in accordance with results previously obtained for asymptotic stretching distributions in some $2 \mathrm{D}$ and $3 \mathrm{D}$ flows $[17,29]$.

For Model 4 we have determined the means $\overline{\log S}$ and standard deviations $\delta$ for the Gaussian distributions illustrated in Fig. 14 as well at other times. At each time $t$ we have 
Fig. 14 (Color online) For Model 4 the PDFs of $\log S$ (green circles $)$ at different times $(t=50$, $100,150,200)$ along with the quantitative Gaussian fit to the data (red curves) are given

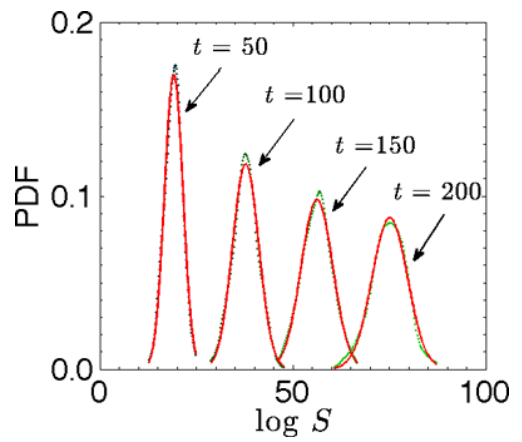

Fig. 15 (Color online) Model 4 $\omega=2.5, \varepsilon=1$ and $b=1$. The coefficients of variation of the Gaussian distributions of $\log S$ at various times $t$ are given. Also included is the best fit of (39) to the data

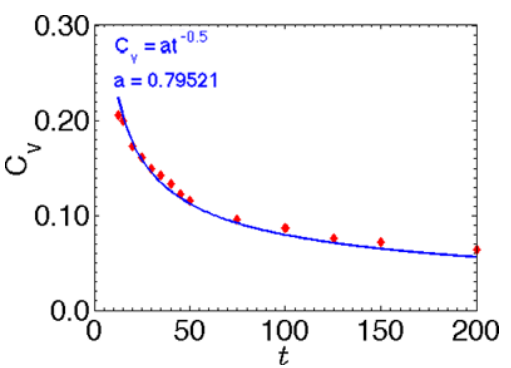

determined the coefficient of variation

$$
\mathrm{C}_{\mathrm{V}}=\frac{\delta}{\overline{\log S}}
$$

The dependence of the coefficient of variation on time is illustrated in Fig. 15. Clearly there is a systematic decrease in $C_{v}$ for increasing times $t$.

Muzzio et al. [25] have hypothesized that for chaotic flows the overall stretching experienced by a tracer is a multiplicative process. Each process can be attributed to the exponential stretching of the tracer at a stagnation point. Thus the cumulative stretching experienced by the tracer after a time $t$ is a product of the stretching experienced during each individual period. Therefore

$$
\mathrm{S}_{0, t}=\mathrm{S}_{0,1} \times \mathrm{S}_{1,2} \times \mathrm{S}_{2,3} \cdots \times \mathrm{S}_{t-1, t}
$$

where $\mathrm{S}_{i-1, i}$ indicates the stretching during the period $t=i-1$ to $t=i$. Thus for long time periods the multipliers $S_{i-1, i}$ act as random numbers. Thus

$$
\log \mathrm{S}_{0, t}=\log \mathrm{S}_{0,1}+\log \mathrm{S}_{1,2}+\log \mathrm{S}_{2,3}+\cdots+\log \mathrm{S}_{t-1, t}
$$

and according to the central limit theorem, on long time scales, $\log \mathrm{S}_{0, t}$ has a Gaussian distribution. Using this approach Muzzio et al. [25] argue that the mean $\overline{\log S}$ and variance $V=\sigma^{2}$ increase linearly with time so that from (36) we have

$$
\mathrm{C}_{\mathrm{V}}=a t^{-\frac{1}{2}}
$$

As can be seen in Fig. 15 this result is in excellent agreement with our simulations. 


\section{Discussion}

We use the cellular flow model described by Solomon and Mezic [34] to study the stretching properties of a three-dimensional, incompressible, unsteady, cellular flow. This particular model has been well studied and allows for independent control of the amplitude of time dependence and strength of the three-dimensional component of the flow. The independent control of these parameters allows for a quantitative study on the effect of these parameters on stretching and hence mixing ability in this model flow. We vary the non dimensional flow parameters $\varepsilon$ (three-dimensional component of velocity field), $\omega$ (oscillation frequency) and $b$ (amplitude of time dependence) to study the stretching characteristics in a suite of models. We present graphs from four of these models. The four models of cellular flow (Table 1), for which we present stretching and the corresponding PDF plots, are different from one another. For $\varepsilon=0.005$ and $b=0.01$, the flow behaves like a vortical flow confined by solid boundaries and the particle paths lie on toric surfaces. However, as the values of $\varepsilon$ and $b$ are increased up to 1.0 to make the flow strongly three-dimensional and time-dependant, the flow looses its vortical structure and the particle paths no longer lie on the toric surfaces.

Comparing our stretching results to that of Solomon and Mezic [34], we find that the computation of cumulative stretching of passive tracers is a good proxy for studying mixing of passive heterogeneities in fluid flows. In Model 1, we see that although the particle path is space filling (Fig. 5(b)) and the mixing is chaotic, the stretching distribution is heterogeneous (Fig. 5(a)). Regions of strong and weak stretching co-exist, creating a wide spectrum of stretching values. This we call weak chaotic mixing. Model 2 exhibits inefficient mixing due to the presence of regular elliptic islands in the flow (Fig. 6(b)), this is reflected as a more heterogeneous stretching distribution compared to Model 1 (Fig. 6(a)). In Model 3 we find that the particle path is space filling (Fig. 7(b)) and the stretching distribution more homogeneous compared to Models 1 and 2 (Fig. 7(a)). This model lies in the transitory region between weak and strong chaotic mixing. In Model 4, we find that the particle path is again space filling (Fig. 8(a)) and the stretching distribution very uniform compared to Models 1, 2 and 3 (Fig. 8(b)). The uniformity of the stretching distributions in Models 1 and 4 can be evaluated by comparing the mean stretching rate, given by 7 , experienced by a arbitrary test tracer with that experienced by the ensemble of 500 tracers. For Model 1 the mean stretching rate for a test tracer starting at $(1.1,0,0)$ is found to be 0.104 , while that for the ensemble of 500 tracers is found to be 0.044 . For Model 4 the mean stretching rate for a test tracer starting at $(1.1,0,0)$ is found to be 0.820 , while that for the ensemble of 500 tracers is found to be 0.860 . Although the test tracer at $(1.1,0,0)$ was chosen arbitrarily, this conclusion would be valid for a tracer starting at any point along $(-0.5,0,0) \times(1.5,0,0)$. This clearly shows that the stretching distribution in Model 4 is more uniform than the stretching distribution in Model 1. This homogeneity of stretching distribution along with the space filling nature of the particle path, we suggest, is a characteristic of strong chaotic mixing.

The PDFs of Models 1 and 2 exhibit two peaks (Fig. 11), a primary peak corresponding to the low end of the stretching spectrum and a secondary peak corresponding to the high end of the stretching spectrum. The primary peak in Model 1 is three orders of magnitude higher than the primary peak in Model 2 and can be attributed to the bulk dynamics of the regions in the flow experiencing weak chaotic mixing in this model. The secondary peak in Model 1 is stronger than that in Model 2 but is four orders of magnitude lower and narrower. This indicates that a larger number of particles undergo moderately high stretching in Model 1 as opposed to fewer particles undergoing a range of high stretching values as in Model 2. In Model 2 the primary peak is representative of the stretching experienced by tracers 

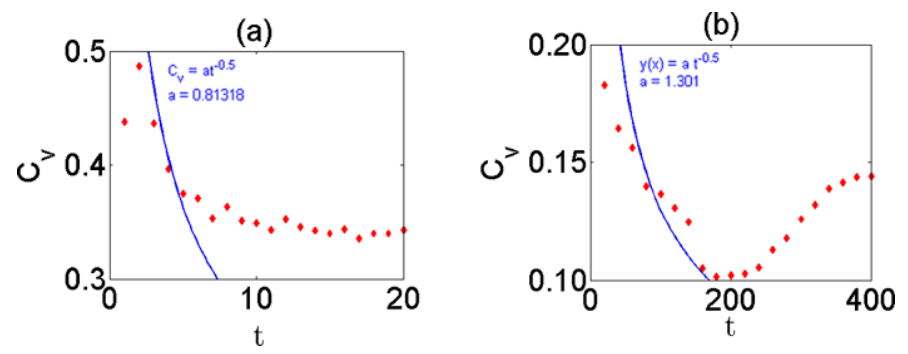

Fig. 16 (Color online) The coefficient of variation of the stretching distribution for the $A B C$ flow and Model 1. (a) Coefficient of variation of the stretching distribution for the $A B C$ flow is shown along with a best fit curve of the form $a t^{-0.5}$. The data is shown for a initial grid of $212 * 212$ tracers starting in the $x=0$ plane. The values of $A, B, C$ are chosen as $\sqrt{3}, \sqrt{2}, 1$ respectively. As can be seen the coefficient of variation decreases significantly slower than the predicted $t^{-0.5}$. This is because the $A B C$ flow does not exhibit global chaos due to the presence of regular KAM tori. (b) The coefficient of variation of the stretching distribution for Model 1 is shown along with the best fit curve of the form $a t^{-0.5}$. After a initial decrease, the coefficient of variation increases again showing that although the mean decreases linearly in time (Fig. 9), the variance of the distribution does not

starting in the excluded, white, regions in the Poincaré map (Fig. 6(b)) and the secondary peak is indicative of the bulk dynamics of the particles starting in the regular elliptic islands. Both Models 3 and 4 do not show secondary peaks. From Figs. 9-13 we also see that the stretching peaks in Models 1 and 2 remain almost stationary in time, while the peaks in Models 3 and 4 migrate towards higher stretching values. This suggests that Model 1 will not obey the random multiplicative process theory for chaotic mixing put forward by Muzzio et al. [25], while in Models 3 and 4 mixing proceeds through a random multiplicative process. This prediction is amply born out in Fig. 16. This difference in the time evolution of the coefficient of variation of stretching values, we suggest, is another difference between weak and strong chaotic mixing.

Based on other simulations, Models 5-13, we find that, for the flow considered in this study, the transition from weak chaotic mixing to strong chaotic mixing is primarily a result of the time dependence of the flow. This is indicated by the increase in the mean stretching rate, $\lambda$, as $b$ is increased (i.e., $\varepsilon$ is held constant; Models 3, 5 to 8). A similar increase is not observed when increasing $\varepsilon$ (i.e., holding $b$ constant; Models 9 to 13) (Table 2). This transition from weak to strong chaotic mixing is accompanied by a change in the topology of the particle paths (Fig. 3). Since even steady flows like the $A B C$ flow [10], a steady solution to the Euler equations, or the STF flow [5], a solution to the Stokes equation, can produce chaotic particle paths it is incorrect to associate chaotic particle paths, and subsequently strong chaotic mixing, with time dependence. As described above, for the vortical flow studied in this paper, this transition from weak to strong chaotic mixing is facilitated by time dependence of the flow, however, this will not be true in general.

Based on our study, we suggest that only those flows which exhibit both global chaos and uniform Lagrangian dynamics (uniform stretching) throughout the flow domain will exhibit strong chaotic mixing. We have already shown that these two properties are satisfied for Model 4, however, to strengthen our conclusion, we present results from a flow that is not globally chaotic, the $A B C$ flow [10], and from a flow that is globally chaotic but does not exhibit uniform Lagrangian dynamics, Model 1.

From Fig. 16 we see that our hypothesis is amply justified, both Model 1 and the $A B C$ flow do not show a power law decrease in the coefficient of variation of the stretching distribution, similar to Model 4. While for the $A B C$ flow this is due to the lack of global chaos 
(regular KAM tori) [10], for Model 1 it is due to the difference in the time required by tracers starting at different points to be space filling (different time scales associated with the Lagrangian dynamics). Suggesting a general route to uniform global chaos based on the current study is not possible. For the flow considered in this paper time dependence is the governing mechanism, however, as mentioned above, this will not be true in general. Although a connection between chaotic advection and chaotic mixing has been established before, a transition from weak to strong chaotic mixing due to the time scales associated Lagrangian dynamics has not been reported before in numerical models of fluid flow.

The cellular flow model developed by Solomon and Mezic [34] captures the important features of vortical flows bounded by rigid boundaries. Since such flows are common in nature, we believe the stretching properties observed here will be widely applicable. In this paper we extend the scope of this model in order to study the effects of moderate and strong three-dimensionality and time dependence on stretching distributions and hence mixing. The flows do not emulate vortical flows in the strongly three-dimensional and time-dependent limit. However, in this limit we believe this study is relevant to three-dimensional, unsteady, and incompressible flows encountered in strongly chaotic systems. Since the spatiotemporal dependence of the velocity field and the structure of the flow seem to be important factors in determining the effectiveness of mixing, we believe this study contributes to the understanding of mixing in many commonly studied model flows.

Acknowledgements We thank M. Billen, M.B. Yikilmaz, and Oliver Kreylos for useful discussions on mixing. We also thank two anonymous reviewers for comments that improved the manuscript.

Open Access This article is distributed under the terms of the Creative Commons Attribution Noncommercial License which permits any noncommercial use, distribution, and reproduction in any medium, provided the original author(s) and source are credited.

\section{References}

1. Anderson, P.D., Galaktionov, O.S., Peters, G.W.M., van deVosse, F.N., Meijer, H.E.H.: Analysis of mixing in three-dimensional time-periodic cavity flows. J. Fluid Mech. 386, 149-166 (1999)

2. Antonsen, T.M., Fan, Z., Ott, E., Garcia-Lopez, E.: The role of chaotic orbits in the determination of power spectra of a passive scalars. Phys. Fluids 8, 3094 (1996)

3. Aref, H.: Stirring by chaotic advection. J. Fluid Mech. 143, 1-21 (1984)

4. Arratia, P.E., Gollub, J.P.: Statistics of stretching fields in experimental fluid flows exhibiting chaotic advection. J. Stat. Phys. 121, 805-822 (2005)

5. Bajer, K., Moffat, H.K.: On a class of steady confined stokes flows with chaotic streamlines. J. Fluid Mech. 212, 337-363 (1990)

6. Bilby, B.A., D Eshelby, J., Kundu, A.K.: The change of shape of a viscous ellipsoidal region embedded in a slowly deforming matrix having a different viscosity. Tectonophysics 28, 265-274 (1975)

7. Chaiken, J., Chevray, R., Tabor, M., Tan, Q.M.: Experimental study of Lagrangian turbulence in a stokes flow. Proc. R. Soc. Lond. A 408, 165-174 (1986)

8. Chien, W.L., Rising, H., Ottino, J.M.: Laminar mixing and chaotic mixing in several cavity flows. J. Fluid Mech. 170, 355-377 (1986)

9. Desmet, G., Verelst, H., Baron, G.V.: Local and global dispersion effects in Taylor-Couette flow-ii. Quantitative measurements and discussion of the reactor performance. Chem. Eng. Sci. 51, 1299-1309 (1996)

10. Dombre, T., Frisch, U., Greene, J.M., Hénon, M., Mehr, A., Soward, A.M.: Chaotic streamlines in the abc flow. J. Fluid Mech. 167, 353-391 (1986)

11. Fountain, G.O., Khakhar, D.V., Mezic, I., Ottino, J.M.: Chaotic mixing in bounded three-dimensional flow. J. Fluid Mech. 417, 265-311 (2001)

12. Fountain, G.O., Khakhar, D.V., Ottino, J.M.: Visualization of three-dimensional chaos. Science 281, 683-686 (1998)

13. Hackborn, W.W., Ulucakli, M.E., Yuster, T.: A theoretical and experimental study of hyperbolic and degenerate mixing regions in a chaotic stokes flow. J. Fluid Mech. 346, 23-48 (1997) 
14. Haller, G.: Response to "comment on finding invariant manifolds in two-dimensional velocity fields". Chaos 11, 431-437 (2001)

15. Haller, G.: Distinguished material surfaces and coherent structures in three-dimensional fluid flows. Physica D 149, 248-277 (2001)

16. Hobbs, D.M., Alvarez, M.M., Muzzio, F.J.: Mixing in globally chaotic flows: a self-similar process. Fractals 5, 395-425 (1997)

17. Hobbs, D.M., Muzzio, F.J.: The kenics static mixer: a three-dimensional chaotic flow. Chem. Eng. J. 67, 153-166 (1997)

18. Jana, S.C., Metcalfe, G., Ottino, J.M.: Experimental and computational studies of mixing in complex stokes flows: the vortex mixing flow and multicellular cavity flows. J. Fluid Mech. 269, 199-246 (1994)

19. Kellogg, L.H., Turcotte, D.L.: Mixing and the distribution of heterogeneities in a chaotically convecting mantle. J. Geophys. Res. 95, 421-432 (1990)

20. Kruijt, P.G.M., Galaktionov, O.S., Anderson, P.D., Peters, G.W.M., Meijer, H.E.H.: Analyzing mixing in periodic flows by distribution matrices: mapping method. AiChE J. 47, 1005-1015 (2001)

21. Kusch, H.A., Ottino, J.M.: Experiments on mixing in continuous chaotic flows. J. Fluid Mech. 236, 319-348 (1992)

22. Lapeyre, G., Hua, B.L., Legras, B.: Comments on “ finding invariant manifolds in two-dimensional velocity fields'. Chaos 11, 427-432 (2001)

23. Lorenz, E.N.: Deterministic nonperiodic flow. J. Atmospheric Sci. 20, 130-141 (1963)

24. McKenzie, D.: Finite deformation during fluid flow. Geophys. J. R. Astron. Soc. 58, 698-715 (1979)

25. Muzzio, F.J., Swanson, P.D., Ottino, J.M.: The statistics of stretching and stirring in chaotic flows. Phys. Fluids A 3, 822-834 (1991)

26. Ottino, J.: The Kinematics of Mixing: Stretching, Chaos and Transport. Cambridge University Press, Cambridge (1989)

27. Ottino, J.M., Jana, S.C., Chakravarthy, V.S.: From Reynolds stretching and folding to mixing studies using horseshoe maps. Phys. Fluids 6, 685 (1994)

28. Ottino, J.M., Leong, C.W., Rising, H., Swanson, P.D.: Morphological structures produced by mixing in chaotic flows. Nature 333, 419-425 (1988)

29. Ottino, J.M., Muzzio, F.J., Tjahjadi, M., Franjione, J.G., Jana, S.C., Kusch, H.A.: Chaos, symmetry and self-similarity: exploiting order and disorder in mixing processes. Science 257, 754-760 (1992)

30. Pattanayak, A.K.: Characterizing the metastable balance between chaos and diffusion. Physica D 148, 1-19 (2001)

31. Rom-Kedar, V., Leonard, A., Wiggins, S.: An analytical study of transport, mixing and chaos in an unsteady vortical flow. J. Fluid Mech. 214, 347-394 (1990)

32. Rudman, M.: Mixing and particle dispersion in the wavy vortex regime of Taylor-Couette flow. AiCE J. 44, 1015-1026 (1997)

33. Shariff, K., Leonard, A., H Ferziger, J.: Dynamical systems analysis of fluid transport in time periodic vortex ring flows. Phys. Fluids 18, 04714 (2006)

34. Solomon, T.H., Mezic, I.: Uniform resonant chaotic mixing in fluid flows. Nature 425, 376-380 (2003)

35. Speetjens, M.F.M., Clercx, H.J.H., Van Heijst, G.J.F.: A numerical and experimental study on advection in three-dimensional stokes flows. J. Fluid Mech. 514, 77-105 (2004)

36. Vainchtein, D.L., Widloski, J., Grigoriev, R.O.: Resonant chaotic mixing in a cellular flow. Phys. Rev. Lett. 99, 094501 (2007)

37. Voth, G., Haller, G., Gollub, J.P.: Experimental measurement of stretching fields in fluid mixing. Phys. Rev. Lett. 88, 254501 (2002)

38. Voth, G., Saint, T.C., Dobler, G., Gollub, J.P.: Mixing rates and symmetry breaking in two-dimensional chaotic flow. Phys. Fluids 15, 2560 (2003)

39. Wereley, S., Lueptow, R.M.: Spatio-temporal character of non-wavy and wavy Taylor-Couette flow. J. Fluid Mech. 364, 59-80 (1998)

40. Wolf, A., Swift, J.B., Swinney, H.L., Vastano, J.A.: Determining Lyapunov exponents from a time series. Physica D 16, 285-317 (1985) 